\title{
Os benefícios da aromaterapia e dos cosméticos orgânicos na recuperação de pacientes com câncer de mama e o papel do enfermeiro
}

The benefits of aromatherapy and organic cosmetics in the recovery of patients with breast cancer and the role of the nurse

Los beneficios de la aromaterapia y la cosmética orgánica en la recuperación de pacientes con cáncer de mama y el papel de la enfermera

Deborah da Silva $^{1 *}$, Maria Cristina Paganini ${ }^{1}$.

\section{RESUMO}

Objetivo: Descrever os benefícios da aromaterapia e os cosméticos orgânicos para pacientes com câncer de mama e a atuação do enfermeiro nas orientações e indicações de uso para a recuperação e tratamento. Métodos: Tratouse de um artigo de revisão de literatura, realizou-se um levantamento nas diferentes bases de dados como: SCIELO, BVS, Google Acadêmico, LILACS, MEDLINE, revistas e sites de órgãos regulatórios, publicados entre 2005 à 2020. Resultados: Foram incluídos 27 artigos no estudo e identificadas 4 categorias distintas, sendo elas: Reações Adversas da Quimioterapia e Radioterapia no Paciente Oncológico; O Câncer de Mama e os Cosméticos; Cosméticos Orgânicos ou Biocosméticos e O Papel do enfermeiro na Recuperação de Pacientes com Câncer de Mama em Uso de Aromaterapia. Considerações finais: A aromaterapia é uma prática que faz parte das Práticas Integrativas Complementares em Saúde (PICS), ainda pouco utilizada no Brasil pelos enfermeiros, porém respaldada pelo COFEN, vem se expandindo e inserindo o enfermeiro nesse cenário, sendo fundamental no sucesso da recuperação da paciente com câncer de mama.

Palavras-chave: Câncer de mama, Cosméticos, Parabenos, Aromaterapia, Quimioterapia.

\section{ABSTRACT}

Objective: To describe the benefits of aromatherapy and organic cosmetics for patients with breast cancer and the role of nurses in the guidelines and indications for use for recovery and treatment. Methods: It was a literature review article, a survey was carried out in different databases such as: SCIELO, BVS, Google Scholar, LILACS, MEDLINE, magazines and websites of regulatory bodies, published between 2005 to 2020 . Results: 27 articles were included in the study and 4 distinct categories were identified, namely: Adverse Reactions from Chemotherapy and Radiotherapy in Oncology Patients; Breast Cancer and Cosmetics; Organic Cosmetics or Biocosmetics and The role of nurses in the recovery of patients with breast cancer using aromatherapy. Final considerations: Aromatherapy is a practice that is part of the Complementary Integrative Health Practices (PICS), still little used in Brazil by nurses, but supported by COFEN, has been expanding and inserting nurses in this scenario, being fundamental in the success of recovery of the breast cancer patient.

Key words: Breast cancer, Cosmetics, Parabens, Aromatherapy, Chemotherapy.

\section{RESUMEN}

Objetivo: Describir los beneficios de la aromaterapia y la cosmética orgánica para pacientes con cáncer de mama y el papel del enfermero en las pautas e indicaciones de uso para la recuperación y el tratamiento. Métodos: Fue un artículo de revisión de la literatura, se realizó una encuesta en diferentes bases de datos como: SCIELO, BVS, Google Scholar, LILACS, MEDLINE, revistas y sitios web de organismos reguladores, publicados entre 2005 a 2020. Resultados: Se incluyeron 27 artículos en el estudio y se identificaron 4 categorías distintas, a saber: reacciones adversas de la quimioterapia y radioterapia en pacientes oncológicos; Cáncer de mama y cosméticos; Cosmética Orgánica o Biocosmética y El papel de las enfermeras en la recuperación de pacientes con cáncer de mama mediante aromaterapia. Consideraciones finales: La aromaterapia es una práctica que forma parte de las Prácticas Complementarias de Salud Integrativa (PICS), aún poco utilizada en Brasil por enfermeras, pero apoyada por COFEN, se ha ido expandiendo e insertando enfermeras en este escenario, siendo fundamental en el éxito de la recuperación. de la paciente con cáncer de mama.

Palabras clave: Cáncer de mama, Productos cosméticos, Parabenos, Aromaterapia, Quimioterapia.

1 Universidade Tuiuti do Paraná (UTP), Curitiba - PR. *E-mail: deborahbee2016@gmail.com 


\section{INTRODUÇÃO}

De acordo com o Instituto Nacional do Câncer (INCA) o câncer de mama é uma patologia crônica caracterizada pela multiplicação desordenada das células formando o tumor, é responsável pela quinta causa de morte por câncer em geral e a primeira causa de morte por câncer em mulheres. No Brasil em 2019 foram 600 mil novos casos de câncer em geral, sendo que 59.700 mil foram casos de câncer mama. Em 2020 a estimativa de novos casos foi de $625.370 \mathrm{com} 66.280$ de casos de mama, esse valor corresponde a um risco estimado de 61,61 casos novos a cada 100 mil mulheres. Os tratamentos contra o câncer de mama são: tratamento local (cirurgia e radioterapia e tratamento sistêmico); quimioterapia, hormonoterapia e terapia biológica (INCA, 2019).

Tanto o tratamento cirúrgico, como o tratamento quimioterápico e radioterápico estão implicados no aparecimento de inúmeros efeitos colaterais. A pele, os cabelos, as unhas e as mucosas são estruturas que podem apresentar alterações decorrentes do estado fisiológico do paciente. Dentre as alterações dermatológicas desencadeadas pelo tratamento oncológico, os mais frequentes são: xerose cutânea, alopecia, dermatite seborreica, dermatite atópica, dermatite de contato, hipercromia local, prurido, radiodermite, melasma, oncomicose, rosácea, eritema, quelóide, alterações da cor da unha, onicólise, queilite (FABRA DG, et al., 2009).

O câncer de mama e seu tratamento podem conduzir a mulher a alterações na sua auto-imagem, perda funcional, alterações psíquicas, emocionais e sociais (MAKLUF DB, et al., 2006). Os principais fatores de risco relacionados ao câncer são: fatores da história reprodutiva e hormonal, fatores genéticos e hereditários, fatores comportamentais e ambientais, e fatores a exposição a determinadas substâncias e ambientes, como agrotóxicos, benzeno, compostos orgânicos voláteis (componentes químicos presentes em diversos tipos de materiais sintéticos ou naturais, hormônios e dioxinas (poluentes orgânicos persistentes altamente tóxicos ao ambiente (INCA 2019).

Outras substâncias ligadas à carcinogênese são os estrógenos e outras substâncias químicas com propriedades estrogênicas também chamadas de xenoestrógenos, que estão associados ao câncer de mama. Os estrógenos desempenham um papel importante na proliferação do epitélio mamário, tanto normal quanto neoplásico. Estudos epidemiológicos, experimentais e clínicos confirmaram a associação entre estrógenos (xenoestrógenos) e câncer de mama,pois eles podem interferir com as funções do sistema endócrino, denominados então dedesreguladores endócrinos, como exemplos temos os pesticidas, conservantes e fragrâncias sintéticas presentes nos cosméticos (PARENTE LML, et al., 2015).

Portanto a aromaterapia é a ciência que visa promover a saúde e o bem-estar do corpo, da mente e das emoções, através do uso terapêutico do aroma natural das plantas por meio de seus óleos essenciais. Essa prática se institucionalizou por meio da Política Pública de Prática Integrativa e Complementar em Saúde (PNPICS), com a portaria no 02/03/2018 efetivado pelo Ministério da Saúde. (SANTOS ACF, et al., 2019).

A Enfermagem, como profissão, reconheceu as PIC de saúde por meio da Resolução no 197 de 1997 do Conselho Federal de Enfermagem (COFEN) como uma especialidade de competência do profissional dessa área, desde que o profissional conclua um curso na área específica, em instituição reconhecida de ensino e com carga horária mínima de 360 horas. Portanto, o enfermeiro tem um papel importante na recuperação do paciente com câncer, conforme o Conselho Federal de Enfermagem, que conceitua a enfermagem como uma ciência e uma arte que fundamenta suas ações na prevenção das doenças, no alívio do sofrimento e na proteção, na promoção e na recuperação da saúde de indivíduos, de famílias, de comunidades e de populações (COFEN, 2020).

Em pacientes com câncer, as PICS são utilizadas em associação com a quimioterapia, tratamento cirúrgico e nos casos clínicos com pior prognóstico, buscando benefícios para os pacientes. O enfermeiro tem grande responsabilidade em fornecer as orientações sobre a possibilidade de utilização dessa prática para proporcionar um cuidado preventivo em relação aos efeitos colaterais dos tratamentos que o paciente com câncer se submete (MENDES DS, et al., 2019). É de suma importância considerar a obtenção do conhecimento sobre os cosméticos convencionais utilizados por esses pacientes e sua toxicidade, bem como 
os benefícios do uso da aromaterapia e os cosméticos orgânicos, como uma prática complementar, especialmente ao paciente oncológico, justificando a necessidade deste estudo. Portanto o estudo objetivouse em descrever os benefícios da aromaterapia e os cosméticos orgânicos para pacientes com câncer de mama e a atuação do enfermeiro nas orientações e indicações de uso para a recuperação e tratamento.

\section{MÉTODOS}

Trata-se de uma revisão de literatura, utilizaram-se artigos consultados em sites de pesquisas como Google Acadêmico, Scientific Electronic Library Online(Scielo),Biblioteca Virtual em Saúde (BVS), Latin American and Caribbean (LILACS), MEDLINE, revistas de Enfermagem, sites de órgãos regulatórios como a Agência nacional de Vigilância sanitária (ANVISA), Conselho Federal de enfermagem (COFEN), INCA, com os seguintes descritores no site DECS: "Câncer de Mama", "Parabenos", "Quimioterapia", "Cosméticos", "Aromaterapia".

O período de busca da revisão foi de quinze anos (2005-2020), foram incluídos materiais em língua portuguesa, disponível na íntegra e excluídos artigos no idioma inglês e espanhol e artigos sem relevância com o tema.

\section{RESULTADOS}

Com o cruzamento dos descritores nas bases de dados foram encontrados 14.446 artigos. Após realizar um refinamento, utilizando os critérios de exclusão e inclusão, foram selecionados e lidos na íntegra 27 artigos, conforme demonstrado na Figura 1.

Figura 1 - Demonstrativo dos materiais selecionados de acordo com a base de dados.

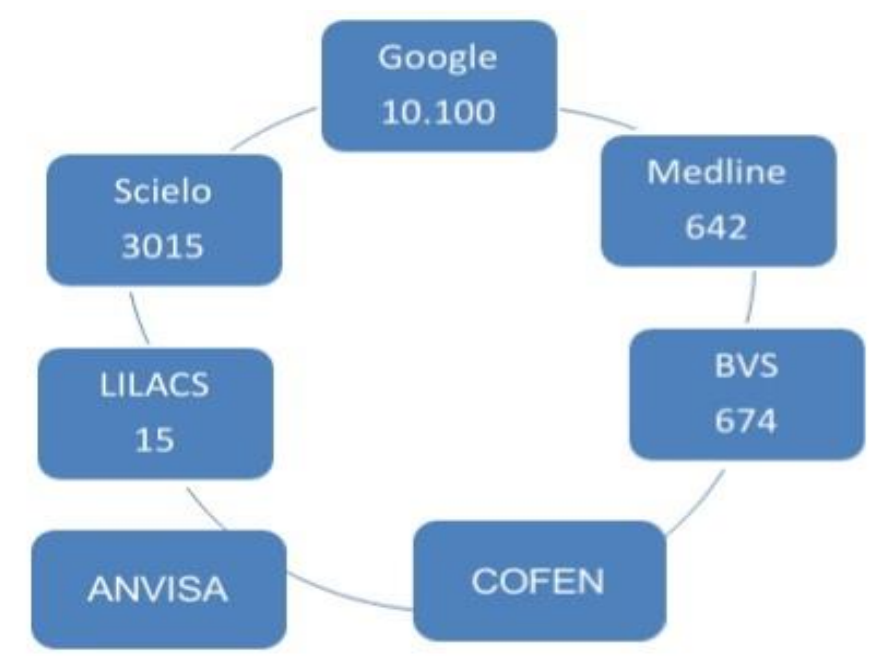

Resultado:

27 artigos

selecionados

Fonte: Silva D e Paganini MC, 2021.

Para uma melhor compreensão dos dados coletados, os artigos foram agrupados de acordo com seu ano de publicação, título, metodologia e objetivo, conforme demonstrado na Tabela 1. 
Tabela 1 - Demonstrativo de artigos selecionados por autor, ano, título, metodologia e objetivos.

\begin{tabular}{|c|c|c|c|}
\hline Autor/Ano & Título & Metodologia & Objetivo \\
\hline $\begin{array}{l}\text { Naziazeno SDS, et al. } \\
\qquad(2020)\end{array}$ & $\begin{array}{l}\text { Diagnósticos de Enfermagem associados á } \\
\text { qualidade de vida de mulheres com câncer de } \\
\text { mama em quimioterapia. }\end{array}$ & Estudo Descritivo & $\begin{array}{l}\text { Identificar diagnósticos de enfermagem em } \\
\text { mulheres com câncer de mama em quimioterapia } \\
\text { por meio do mapeamento cruzado dos problemas } \\
\text { de saúde com a taxonomia NANDA-I }\end{array}$ \\
\hline $\begin{array}{l}\text { Santos ACF, et al. } \\
\qquad(2019)\end{array}$ & $\begin{array}{l}\text { Aromaterapia: uma nova tecnologia de cuidado, } \\
\text { prevenção e promoção da saúde }\end{array}$ & Revisão de literatura & $\begin{array}{l}\text { Averiguar o processo de validação da } \\
\text { Aromaterapia dentro da PNPICS e do contexto do } \\
\text { SUS. }\end{array}$ \\
\hline Mendes DS, et al. (2019) & $\begin{array}{l}\text { Benefícios das práticas integrativas e } \\
\text { complementares no cuidado de enfermagem }\end{array}$ & Revisão de literatura & $\begin{array}{l}\text { Caracterizar os benefícios das práticas } \\
\text { integrativas e complementares no cuidado de } \\
\text { enfermagem }\end{array}$ \\
\hline Costa CC, et al. (2019) & $\begin{array}{l}\text { Radiodermatites: Análise dos Fatores Preditivos } \\
\text { em Pacientes com Câncer de Mama }\end{array}$ & Estudo Retrospectivo & $\begin{array}{l}\text { Avaliar os fatores associados com o aparecimento } \\
\text { de radiodermite após radioterapia e a sua } \\
\text { associação com o maior grau de toxicidade nesses } \\
\text { pacientes }\end{array}$ \\
\hline Silva PR, et al. (2019) & $\begin{array}{l}\text { Qualidade do sono e fadiga em mulheres com } \\
\text { câncer de mama durante o tratamento } \\
\text { quimioterápico. }\end{array}$ & Estudo Descritivo & $\begin{array}{l}\text { Verificar a ocorrência de alterações do sono, antes } \\
\text { e após o tratamento quimioterápico. }\end{array}$ \\
\hline Magalhães LS (2018) & $\begin{array}{l}\text { Cosméticos Orgânicos, uma tendência crescente } \\
\text { no mercado. }\end{array}$ & Revisão de literatura & $\begin{array}{l}\text { Proporcionar maior conhecimento sobre } \\
\text { mercado dos cosméticos orgânicos }\end{array}$ \\
\hline Romero V, et al. (2018) & $\begin{array}{l}\text { Diferenças entre cosméticos orgânicos e naturais: } \\
\text { literatura esclarecedora para Prescritores }\end{array}$ & Revisão de literatura & $\begin{array}{l}\text { Realizar revisão sistematizada da literatura sobre } \\
\text { legislações e publicações sobre cosméticos } \\
\text { orgânicos e naturais }\end{array}$ \\
\hline Silva CB, et al. (2017) & $\begin{array}{l}\text { A importância da ação antioxidante de óleos } \\
\text { essenciais em benefício da saúde }\end{array}$ & Revisão de literatura & $\begin{array}{l}\text { Realizar um levantamento acerca da importância } \\
\text { da ação antioxidante presente nos óleos } \\
\text { essenciais na promoção ao benefício da saúde. }\end{array}$ \\
\hline Hoppe AC, et al. (2017) & $\begin{array}{l}\text { Avaliação da toxicidade de parabenos em } \\
\text { cosméticos }\end{array}$ & Revisão de literatura & $\begin{array}{l}\text { Avaliar o risco quanto à utilização de cosméticos e } \\
\text { produtos de higiene que contenham parabenos } \\
\text { como conservante. }\end{array}$ \\
\hline
\end{tabular}




\begin{tabular}{|c|c|c|c|}
\hline Autor/Ano & Título & Metodologia & Objetivo \\
\hline Gnatta JR, et al. (2016) & $\begin{array}{l}\text { Aromaterapia e enfermagem: concepção histórico- } \\
\text { teórica. Revista da Escola de Enfermagem }\end{array}$ & Estudo Descritivo & $\begin{array}{l}\text { Discorrer sobre a farmacognosia dos óleos } \\
\text { essenciais, a trajetória histórica da Aromaterapia } \\
\text { na Enfermagem }\end{array}$ \\
\hline Vieira RAC, et al. (2016) & $\begin{array}{l}\text { Instrumentos da avaliação quantitativa e } \\
\text { qualitativa das sequelas relacionadas ao } \\
\text { tratamento do câncer de mama }\end{array}$ & Revisão de literatura & $\begin{array}{l}\text { Apresentar os principais métodos de avaliação das } \\
\text { sequelas relacionadas ao tratamento de câncer de } \\
\text { mama. }\end{array}$ \\
\hline $\begin{array}{l}\text { Guimarães RCR, et al. } \\
(2015)\end{array}$ & $\begin{array}{l}\text { Ações de enfermagem frente as reações a } \\
\text { quimioterápicos em pacientes oncológicos. }\end{array}$ & Revisão de literatura & $\begin{array}{l}\text { Descrever as ações de enfermagem frente as } \\
\text { reações a quimioterápicos em pacientes } \\
\text { oncológicos. }\end{array}$ \\
\hline $\begin{array}{l}\text { Parente LML, et al. } \\
\qquad(2015)\end{array}$ & Câncer de mama e cosméticos & Revisão de literatura & $\begin{array}{l}\text { Abordar os efeitos carcinogênicos de cosméticos } \\
\text { em relação ao câncer de mama. }\end{array}$ \\
\hline Souza AB, et al. (2014) & $\begin{array}{l}\text { O uso do antitranspirante e suas reações } \\
\text { adversas. Revista Eletrônica Biociência, } \\
\text { Biotecnologia e Saúde. }\end{array}$ & Revisão de literatura & $\begin{array}{l}\text { Apresentar os efeitos adversos que o uso rotineiro } \\
\text { dos antiperspirantes pode causar ao organismo }\end{array}$ \\
\hline $\begin{array}{l}\text { Paganini T, Silva VF. } \\
\qquad(2014)\end{array}$ & O uso da aromaterapia no combate ao estresse. & Revisão de literatura & $\begin{array}{l}\text { Levantar a bibliografia da utilização dos óleos } \\
\text { essenciais no tratamento do estresse. }\end{array}$ \\
\hline Claro F, et al. (2013) & $\begin{array}{l}\text { Complicações em reconstrução mamária total em } \\
\text { pacientes mastectomizadas por câncer de mama: } \\
\text { análise comparativa de longo prazo quanto a } \\
\text { influência de técnica, tempo de cirurgia, momento } \\
\text { da reconstrução e tratamento adjuvante }\end{array}$ & Estudo Observacional & $\begin{array}{l}\text { Identificar as complicações mais frequentes } \\
\text { apresentadas pelas principais técnicas de } \\
\text { reconstrução mamária e compará-las a relevantes } \\
\text { variáveis independentes }\end{array}$ \\
\hline Brito AMG, et al. (2013) & Aromaterapia: da gênese a atualidade & Revisão de Literatura & $\begin{array}{l}\text { Acentuar cada vez mais a curiosidade e o } \\
\text { conhecimento sobre essa arte e ciência milenar, } \\
\text { que se apoia no uso racional dos óleos essenciais. }\end{array}$ \\
\hline Vinas PJR, et al. (2013) & $\begin{array}{l}\text { Os cosméticos podem provocar câncer de mama, } \\
\text { doença de Alzeimer, dores articulares, crises de } \\
\text { asma, puberdade precoce, entre outras patologias }\end{array}$ & Revisão de literatura & Identificar os malefícios dos cosméticos \\
\hline
\end{tabular}




\begin{tabular}{|c|c|c|c|}
\hline Autor/Ano & Título & Metodologia & Objetivo \\
\hline Tozzo M, et al. (2012) & $\begin{array}{l}\text { Biocosméticos ou cosmético orgânico: Revisão de } \\
\text { literatura }\end{array}$ & Revisão de literatura & $\begin{array}{l}\text { Revisar parte da literatura sobre biocosméticos } \\
\text { certificados e disponíveis no mercado brasileiro }\end{array}$ \\
\hline $\begin{array}{l}\text { Cassarotti ALS, et al. } \\
\qquad(2012)\end{array}$ & $\begin{array}{l}\text { Malefícios decorrentes ao uso de produtos } \\
\text { contendo Parabenos. }\end{array}$ & Revisão de literatura & $\begin{array}{l}\text { Avaliar o potencial maléfico dos parabenos aos } \\
\text { seus consumidores. }\end{array}$ \\
\hline Gnatta JR, et al. (2011) & O uso da aromaterapia no alívio da ansiedade & Estudo Descritivo & $\begin{array}{l}\text { Verificar como o uso dos óleos essenciais (OE) de } \\
\text { Lavanda e Gerânio alteram a percepção de } \\
\text { ansiedade e comparar sua eficácia }\end{array}$ \\
\hline Tavares AT, et al. (2011) & $\begin{array}{l}\text { Relação do uso de parabenos em cosméticos e a } \\
\text { sua ação estrogênica na indução do câncer no } \\
\text { tecido mamário. }\end{array}$ & Revisão de literatura & $\begin{array}{l}\text { Discutir a ação estrogênica dos parabenos } \\
\text { presentes nas formulações de cosméticos } \\
\text { utilizados na região do tórax e axilas e sua relação } \\
\text { com o } \\
\text { câncer de mama. }\end{array}$ \\
\hline Criado PR, et al. (2010) & $\begin{array}{l}\text { Reações tegumentares adversas relacionadas aos } \\
\text { agentes antineoplásicos: parte II. }\end{array}$ & Estudo Descritivo & $\begin{array}{l}\text { Expor as informações sobre reações cutâneas à } \\
\text { quimioterapia }\end{array}$ \\
\hline Fabra DG, et al (2009) & $\begin{array}{l}\text { Alterações dermatológicas em pacientes } \\
\text { oncológicos - adultos e crianças }\end{array}$ & Estudo descritivo & $\begin{array}{l}\text { Dividir as alterações dermatológicas e } \\
\text { cosmiátricas } \\
\text { oncológico }\end{array}$ \\
\hline Makluf DB, et al. (2006) & $\begin{array}{l}\text { Avaliação da qualidade de vida em mulheres com } \\
\text { câncer de mama }\end{array}$ & Estudo descritivo & $\begin{array}{l}\text { Avaliar a qualidade de vida em mulheres com } \\
\text { câncer de mama }\end{array}$ \\
\hline Maluf MFM, et al. (2005) & O impacto psicológico do câncer de mama & Revisão de literatura & $\begin{array}{l}\text { Promover o conhecimento do impacto psicológico } \\
\text { produzido pelo câncer de mama através da } \\
\text { revisão da literatura internacional. }\end{array}$ \\
\hline Guimarães JRPF (2005) & $\begin{array}{l}\text { Disruptores endócrinos no meio ambiente: um } \\
\text { problema de saúde pública e ocupacional }\end{array}$ & Revisão de literatura & $\begin{array}{l}\text { Mostrar os conceitos destas substâncias químicas } \\
\text { e seus efeitos na saúde humana, }\end{array}$ \\
\hline
\end{tabular}

Fonte: Silva D e Paganini MC, 2021. 


\section{DISCUSSÃO}

\section{Reações adversas da quimioterapia e radioterapia no paciente oncológico}

Quando se trata de qualidade de vida, de acordo Makluf DB, et al. (2006), existem algumas ferramentas para se avaliar a paciente com câncer de mama, embora o interesse por esse conceito ainda seja recente, 0 assunto está mais frequente nas últimas décadas. O câncer de mama é tratado como uma doença sistêmica e muitas vezes após o tratamento cirúrgico é seguido de uma terapia quimioterápica. Os tratamentos impactam negativamente a vida da paciente, afetando suas dimensões física, psíquica e social.

O impacto psicológico é causado com a confirmação do diagnóstico, conforme descrevem Maluf MFM, et al. (2005), pois a paciente passa pelo processo de luto durante o tratamento por ser portadora da doença, pelo tratamento, pela cirurgia, pela perda da imagem, devido as mudanças corpóreas como a alopecia (queda de cabelo), e pelas possíveis sequelas, gerando a ansiedade, medo, incertezas, e distúrbios psicológicos como a depressão.

Vieira RAC, et al. (2016), afirmam que ferramentas de avaliação das sequelas relacionadas ao câncer de mama são pouco utilizadas, porém é de extrema importância para mensurar e aprimorar os resultados e tratamentos.

No estudo de Costa CC, et al. (2019) analisaram a radioterapia, e observaram que ela provoca uma toxicidade cutânea, conhecida como radio dermatite, que são lesões definidas como um conjunto de reações cutâneas decorrentes da destruição de células basais da epiderme, causando descamação, ulceração, dor, e até mesmo necrose. Quanto ao manejo da radiodermatite, ainda existem controversas, porém há recomendações sobre o uso de chá de camomila, loção a base de ácidos graxos, aloe vera, e a calêndula officialis que são fitoterápicos.

A respeito das alterações dermatológicas em pacientes com câncer Fabral DG, et al (2009) relataram 59 tipos de dermatopatias decorrente aos tratamentos, entre elas a xerose cutânea, caracterizada pela pele mais áspera, opaca, sensível, avermelhada, descamada, pruriginosa, rachada e sujeita a infecção. Criado PR, et al. (2010) descrevem a interação da pele com os medicamentos, e apontam também alterações vasculares, e outras reações adversas como: estomatite, vasodilatação como o flushing, reações autoimunes como lúpus eritematoso, maceração nas áreas das axilas, hiperpigmentação, erupção, acne, psoríase, entre outros.

No tratamento cirúrgico de reconstrução mamária Claro F, et al. (2013), demostraram complicações com diferentes graus de morbidade, dentre elas as complicações no pós-operatório citando a seroma, infecção, contratura, abaulamento abdominal, hérnias e necrose.

\section{$O$ câncer de mama e os cosméticos}

A Agência Nacional de Vigilância Sanitária (ANVISA) define os produtos cosméticos como preparações constituídas por substâncias naturais ou sintéticas, de uso externo nas diversas partes do corpo humano (pele, sistema capilar, unhas, lábios, órgãos genitais externos, dentes e membranas mucosas da cavidade oral) com o objetivo de limpá-los, perfumá-los, alterar sua aparência, corrigir odores corporais e mantê-los em bom estado.

De acordo com Vinas PJR, et al. (2013), as substâncias mais utilizadas nos cosméticos são: parabenos, triclosan, alumínio, silicone, derivados do petróleo como o óleo mineral e a vaselina, polietilenoglicol (PEG) e o formaldeído que atualmente é proibido em vários países. Outras substâncias dos cosméticos foram estudadas por Guimarães JRPF (2005), evidenciando a toxicidade ao organismo humano sendo elas: Chumbo, Ftalatos, Bisfenol. Elas agem por mecanismo fisiológicos pelos quais substituem os hormônios do nosso corpo, bloqueiam a sua ação natural ou aumentam ou diminuem a quantidade natural hormonal alterando as suas funções com efeito acumulativo no organismo.

Cassatori ALS, et al. (2012), afirmam que os parabenos são utilizados em cosméticos, fármacos e alimentos, sendo os mais frequentes: metilparabeno, etilparabeno, benzilparabeno e butilparabeno. Os parabenos são absorvidos pelo trato gastrointestinal e pelo sangue, hidrolisados e excretados pela urina, mas podem permanecer intactos no organismo e acumular nos tecidos do corpo, causando malefícios. A legislação 
brasileira permite o uso de parabenos em produtos cosméticos de até $0,4 \%$, esses conservantes são desreguladores endócrinos, que apresentam atividade estrogênica, uma vez que o estrogênio é o maior fator de crescimento e desenvolvimento de casos de câncer em seres humanos, causam reações adversas, hipersensibilidades, e ainda conforme o autor, as reações causadas pelos cosméticos podem ser dividias por: reações imediatas ou acumulativas; reações alérgicas ou sensibilizantes, que é caracterizada por irritação, sensação de dor, vermelhidão, prurido, essas reações causadas também incluem alteração no sistema reprodutor e aumento de incidência de câncer de mama, devido ao constante uso desse conservante aplicado nas axilas pelo uso de desodorantes.

Hoppe AC, et al. (2017) avaliaram a toxicocinética dos parabenos em cosméticos, concluindo que crianças também sofrem exposição, além de apresentar atividade estrogênica, há outros efeitos tóxicos dos parabenos, como as reações alérgicas no local da aplicação ou até em outra parte do corpo, como a presença de eritema e edema, enfatizando que cosméticos com esses conservantes não são seguros principalmente para a pele.

De acordo com a Tavares AT, et al. (2011), os parabenos podem ser absorvidos pela pele por ter um baixo peso molecular, constataram que $30 \%$ do total de propilparabeno aplicado sobre a pele, consegue penetrar no estrato córneo de forma inalterada. Foram encontrados ésteres intactos em tecido retirado de mama, sugerindo acúmulo dessa substância no tecido.

Segundo Parente LML, et al. (2015), nos cosméticos convencionais, os principais componentes sintéticos xenoestrógenos são os sais de alumínio (presentes em antiperspirantes, triclosan (desodorantes), fragrâncias sintéticas, parabenos (conservantes), mesmo considerando que existam apenas dois estudos epidemiológicos que relacionam diretamente o uso de desodorantes nas axilas a ocorrência do câncer de mama.

Souza AB, et al. (2014) avaliaram o uso de antiperspirante apontando o alumínio e seus derivados, triclosan, parabenos, e seus efeitos relacionados ao uso diário como: reações alérgicas, hidradenite uma inflamação crônica e supurativa das glândulas apócrinas, devido a oclusão folicular. Em relação ao câncer de mama, ainda é bastante discutida, pois a mama é exposta ao alumínio através desses produtos. Porém o uso em longo prazo aumenta a dosagem de alumínio no sangue, que foram associados à dor óssea, fadiga crônica, após a suspensão do uso o nível no sangue baixou e os sintomas desapareceram.

\section{Cosméticos orgânicos ou biocosméticos}

Segundo Tozzo M, et al. (2012) o conceito dos cosméticos orgânicos surgiram desde 1970, onde a cosmetologia ingressou nos movimentos sociais e científicos, se aliando para discutir a respeito da crise ambiental e recomendar o uso de produtos que não causassem danos ao meio ambiente e a saúde humana. Com isso surgiram os biocosméticos ou cosméticos orgânicos que são produtos naturais, com $95 \%$ de matéria prima orgânica, livre de conservantes sintéticos como os parabenos, derivado de animais e não testados em animais. Possuem a capacidade natural de estimular a recuperação da pele, contém ingredientes vegetais, com isso espera-se que eles sejam mais delicados com a pele e cabelos.

Magalhães LS (2018) relata que os cosméticos orgânicos são muito promissores, porém ainda poucos conhecidos no Brasil, trazem inúmeros benefícios, um deles é a recuperação da pele, e diminuição de surgimento de alergias, doenças e toxicidade.

Os cosméticos, segundo Romero V, et al. (2018), estão ganhando cada vez mais espaço no Brasil, por não conter pesticidas, testes em animais, e ser sustentável. A conscientização da sociedade sobre o meio ambiente também contribui com esse crescimento.

Atualmente um dos ingredientes desses cosméticos vem se destacando como uma fonte promissora de ação oxidante e neutralizantes que de acordo com Silva CB, et al. (2017) são benéficos contra o envelhecimento da pele, doenças cardiovasculares e atuam contra algumas espécies radicalares que causam o câncer, são chamados de óleos essenciais.

Os óleos essenciais são utilizados na Aromaterapia, que conceitua em uma terapia baseada no uso de concentrados voláteis extraído de plantas, com a finalidade de melhorar o bem-estar físico, mental e 
emocional, esses óleos, segundo Gnatta JR, et al. (2011), são compostos por moléculas de alta complexidade que podem atuar de diversas maneiras no organismo aplicando por via cutânea ou inalatória. É considerada uma nova ferramenta ao enfermeiro, pois faz parte da Práticas Integrativas e Complementares (PICS) regulamentada pelo COFEN № 197/1997.

\section{O papel do enfermeiro na recuperação de pacientes com câncer de mama em uso de aromaterapia}

A quimioterapia é uma das modalidades mais empregadas no controle do câncer, porém seus eventos adversos, segundo Silva PR, et al. (2019), afetam as esferas biopsicossociais e geram alterações e desconfortos como as náuseas, vômitos, distúrbios do sono e fadiga. No manejo dessas alterações a atuação do enfermeiro é de grande valia, pois a identificação desses sintomas é necessária para promoção do alívio e manejo de tais eventos adversos.

De acordo com Naziazeno SDS, et al. (2020) na prática clínica, os diagnósticos de enfermagem identificados podem subsidiar a consulta e a assistência de enfermagem à mulher com câncer de mama. Alguns diagnósticos apresentados nesse estudo foram: ansiedade, medo, insônia, fadiga, dor aguda, dor crônica, risco de depressão, risco de mucosa oral prejudicada, síndrome do estresse por mudança, diminuição do autocuidado, risco de isolamento social, padrão de sexualidade ineficaz.

Guimarães RCR, et al. (2015) afirmam que a assistência qualificada é um fator determinante para a recuperação bio-psico-socio-espiritual do paciente oncológico, pois querer do enfermeiro multiplicidade de conhecimento e versatilidade na atuação, de forma planejada, sistematizada e bem implementada.

Para subsidiar essa recuperação citada por Guimarães RCR, et al. (2015), os autores Brito AMG, et al. (2013) citam a aromaterapia como uma arte e uma ciência que visa promover a saúde e o bem-estar do corpo, da mente e das emoções, através do uso terapêutico do aroma natural das plantas por meio dos seus óleos essenciais É uma prática muito antiga e atualmente é reconhecida em muitos países industrializados, como um método extremamente eficaz e terapêutico. Alguns óleos essenciais já estão sendo utilizados em substituição de determinados antibióticos com êxito e sem efeitos adversos, já em infecções bacterianas causadas por bactérias Gram negativas, é muito utilizado o óleo essencial de Melaleuca alternifólia, ele apresenta atividade antibacteriana contra Staphylococcus aureus e Bacillus subtillis.

A Aromaterapia de acordo Gnatta JR, et al. (2016) tem sido praticada por Enfermeiros e respalda no Brasil pelo COFEN, auxilia e estabelece o reequilíbrio tanto emocional quanto físico do indivíduo, sendo uma possibilidade de intervenção, representando a aplicação das teorias na prática assistencial. Florence Nighgale foi a primeira enfermeira a usar os óleos essenciais, e a primeira Aromaterapeuta enfermeira foi Marguerite Maury na França abrindo sua clínica com prescrições terapêuticas individualizadas, uma vez que tal prática proporcionam ação física, psíquica, e emocional.

Paganini T e Silva VF (2014) afirmam que o estresse é um dos fatores que mais atingem as pessoas no Brasil, seja por mudanças nos estilos de vida ou outros fatores, os óleos essenciais quando utilizados por profissionais habilitados, proporcionam uma melhora significativa com óleos essenciais com efeitos sedativos, calmantes e relaxantes óleos, tais como: Anis, Alcaravia, Bergamota, Camonila-Romana, Cedro, Cipestre, Erva-cidreira, Lavanda, Gerânio, Sálvia Esclarea, entre outros.

Segundo Santos ACF, et al. (2019) a aromaterapia se institucionalizou por meio da PNPICS, com a portaria no 702/03/2018 efetivada pelo Ministério da Saúde, que busca estar em conformidade com as diretrizes da Organização Mundial da Saúde (OMS).

A Aromaterapia está dentre as Práticas Integrativas e Complementares em Saúde (PICS). De acordo com o Ministério da Saúde do Brasil essas práticas são tratamentos que utilizam recursos terapêuticos baseados em conhecimentos tradicionais, voltados para a prevenção de doenças como a depressão, hipertensão, além de serem usadas para tratamento complementar em diversas Doenças Crônicas Não Transmissíveis (DCNT). Essas práticas de acordo com Paganini T e Silva VF (2014), foi inserida como uma estratégia de atenção e cuidado no SUS, buscando estimular mecanismos naturais de prevenção de agravos e recuperação da saúde.

Foram aprovadas no SUS em 2006 apenas 3 práticas, em 2017 foram acrescentadas 14 e em 2018, foram aprovadas mais 12 novas totalizando 29 PICS. Internacionalmente a OMS reconhece essas práticas como 
uma forma de tratamento, Mendes DS, et al. (2019), dentre as práticas podemos citar a aromaterapia que foi efetivada pelo Ministério da Saúde (BRASIL) dentro da PICS em 2018 pela portaria no 702/03/2018. As PICS podem ser aplicadas como complemento no alívio de vários sintomas, promovendo um cuidado diferenciado e com resultados efetivos, inclusive para pacientes oncológicos em associação com a quimioterapia, tratamento cirúrgico, chegando até em cuidados paliativos, reduzindo, estresse, dor, angústia, ansiedade.

\section{CONSIDERAÇÕES FINAIS}

A aromaterapia é uma prática que faz parte das Práticas Integrativas Complementares em Saúde (PICS), ainda pouco utilizada no Brasil pelos enfermeiros, porém respaldada pelo COFEN, vem se expandindo e inserindo o enfermeiro nesse cenário como de papel fundamental no sucesso da recuperação da paciente com câncer de mama, identificando os diagnósticos de enfermagem e suas intervenções, orientando, e prescrevendo tratamentos coadjuvantes no manejo das diversas reações causadas pelo tratamento. Enfatizase a importância do preparo desse profissional, pois faz-se necessário para a prática aromaterapia bem como o conhecimento da cosmetologia orgânica a fim de obter a autonomia e a aplicabilidade de maneira segura no contexto da assistência da enfermagem.

\section{REFERÊNCIAS}

1. ANVISA - AGÊNCIA NACIONAL DE VIGILÂNCIA SANITÁRIA. RDC № 211, de 14 de julho de 2005. Estabelece a Definição e a Classificação de Produtos de Higiene Pessoal, Cosméticos e Perfumes, conforme Anexo I e II desta Resolução e dá outras definições. Disponível em: http://bvsms.saude.gov.br/bvs/saudelegis/anvisa/2005/rdc0211_14_07_2005.html\#: :text=adota\%2C\%20\%E2\%80 \%9Cad\%20referendum\%E2\%80\%9D\%2C,1\%20e\%20II\%20desta\%20Resolu\%C3\%A7\%C3\%A3o. Acessado em: 12 de fevereiro de 2021.

2. BRITO AMG, et al. Aromaterapia: da gênese a atualidade. Revista Bras de Plantas Medicinais, 2013;15(4): 789-793.

3. CASSAROTTI ALS, et al. Malefícios decorrentes ao uso de produtos contendo Parabenos. Universidade Tuiuti do Paraná, 2012, 1(4): 01-09,

4. CLARO F, et al. Complicações em reconstrução mamária total em pacientes mastectomizadas por câncer de mama: análise comparativa de longo prazo quanto a influência de técnica, tempo de cirurgia, momento da reconstrução e tratamento adjuvante. Rev. Bras. Cir. Plást, 2013; 28(1): 01-10.

5. COFEN- CONSELHO FEDERAL DE ENFERMAGEM. RESOLUÇÃO COFEN № 626/2020. Disponível em: http://www.cofen.gov.br/resolucao-cofen-no-626-2020_77398.html. Acessado em 12 de fevereiro de 2021.

6. COSTA CC, et al. Radiodermatites: Análise dos Fatores Preditivos em Pacientes com Câncer de Mama. Revista Brasileira de Cancerologia, 2019; 65 (1): e-05275.

7. CRIADO PR, et al. Reações tegumentares adversas relacionadas aos agentes antineoplásicos. Revista An. Bras. Dermato, 2010; 85(5): 591-608.

8. FABRA DG, et al. Alterações dermatológicas em pacientes oncológicos - adultos e crianças. Revista Arq. Bras. Ciên. Saúde, 2009; 34(.2):87-93.

9. GNATTA JR, et al. Aromaterapia e enfermagem: concepção histórico-teórica. Revista da Escola de Enfermagem, 2016; 50 (1): 130-136.

10. GNATTA JR. et al. O uso da aromaterapia na melhora de autoestima. Revista Acta Paulista de Enfermagem, 2011; 24 (2): 03-15.

11. GUIMARÃES JRPF. Disruptores endócrinos no meio ambiente: um problema de saúde pública e ocupacional, 2005; 1(2): 01-09.

12. GUIMARÃES RCR, et al. Ações de enfermagem frente às reações e quimioterápicos em pacientes oncológicos. Revista Cuidado é Fundamental, 2015; 7(2): 2440-2452.

13. HOPPEAC, et al. Avaliação da toxicidade de parabenos em cosméticos. Revista Revinter, $2017 ; 10$ (3): $49-70$.

14. INCA - INSTITUTO NACIONAL DE CÂNCER. Números de Câncer no Brasil. 2019. Disponível em: https://www.inca.gov.br/numeros-de-cancer. Acessado em 12 de março de 2021.

15. MAGALHÃES LS. Cosméticos Orgânicos, uma tendência crescente no mercado. Universidade Federal de Ouro Preto, 2018: 01-29.

16. MAKLUF DB, et al. Avaliação da qualidade de vida em mulheres com câncer de mama, Revista Brasileira de Cancerologia. 2006; 52(1): 49-58.

17. MALUF MFM, et al. O impacto psicológico do câncer de mama. Revista de Cancerologia, 2005; 51(2): $149-154$.

18. MENDES DS, et al. Benefícios das práticas integrativas e complementares no cuidado de enfermagem. Journal Health NPEPS, 2019; 4(1): 302-318.

19. NAZIAZENO SDS, et al. Diagnósticos de Enfermagem associados a qualidade de vida de mulheres com câncer de mama em quimioterapia. Revista Cuidado é fundamental, 2020:629-635. 
20. PAGANINI T, SILVA VF. O uso da aromaterapia no combate ao estresse. Revista Arq. Ciênc. Saúde Unipar, 2014; 18(1): 43-49.

21. PARENTE LML, et al. Câncer de mama e cosméticos. Revista Arte Médica Ampliada, 2015; 35(1): 20-23.

22. ROMERO V, et al. Diferenças entre cosméticos orgânicos e naturais: literatura esclarecedora para Prescritores. Universidade de Campinas. 2018; 10(3): 188-193.

23. SANTOS ACF, et al. Aromaterapia: uma nova tecnologia de cuidado, prevenção e promoção da saúde. Revista Científica, 2019; 19(39): 01-22.

24. SILVA CB, et al. A importância da ação antioxidante de óleos essenciais em benefício da saúde. Diversitas Journal, 2017; 2(1): 52-55.

25. SILVA PR, et al. Qualidade do sono e fadiga em mulheres com câncer de mama durante o tratamento quimioterápico. Rev. Enferm. UFSM, 2019; 9(20): 01-18.

26. SOUZA AB. et al. O uso do antitranspirante e suas reações adversas. Revista Eletrônica Biociência, Biotecnologia e Saúde, 2014; 1(10): 01-11.

27. TAVARES AT, et al. Relação do uso de parabenos em cosméticos e a sua ação estrogênica na indução do câncer no tecido mamário. Revista Multidisciplinar da Saúde, 2011;3(6):01-14.

28. TOZZO M, et al. Biocosméticos ou cosmético orgânico: Revisão de literatura. Revista Thêma et Scientia, 2012; 2(1): 122-130.

29. VIEIRA RAC, et al. Instrumentos da avaliação quantitativa e qualitativa das sequelas relacionadas ao tratamento do câncer de mama. Revista Brasileira de Mastologia, 2016; 26(3): 126-132.

30. VINAS PJR, et al. Os cosméticos podem provocar câncer de mama, doença de Alzheimer, dores articulares, crises de asma, puberdade precoce, entre outras patologias. Medicina Complementar, 2013; 1(2): 01-04. 